\title{
Basic study on Transepidermal Water Loss (TEWL) of infants living in urban and non-urban areas and their environmental factors.
}

\author{
Satomi Ono ${ }^{1 *}$, Yukiko Manabe ${ }^{2}$ \\ ${ }^{1}$ Department of Nursing, Graduate School of Health Science, Okayama University, Japan \\ ${ }^{2}$ Former College of Nursing, St. Luke's International University Japan
}

\begin{abstract}
The aim of this study is to document Transepidermal Water Loss (TEWL) in infants living in urban and non-urban areas as well as individual and environmental factors, and to search for any distinguishing features and correlations. A significant difference was observed in trans epidermal water loss in children residing in a region located outside a large city or urban area, and a significant difference was observed also in cuticle water content measured at the same time. It was clear that infants living in an administrative district outside of an urban area retained more water in their cuticles than infants residing in a large city, and transpired more water through the epidermis. It was suggested that TEWL in preschool children may differ according to age and gender, and is influenced by outdoor humidity, outdoor temperature, surface temperature, and cuticle water content.
\end{abstract}

Keywords: Transepidermal Water Loss (TEWL), Infant, Environmental factor, SC water content, Skin's barrier function.

Accepted on May 29, 2017

\section{Introduction}

Transepidermal water loss (hereinafter 'TEWL') is one of the indicators of the skin's barrier function in intercellular lipid of the stratum corneum (SC). In the United States, research about the SC's barrier function rapidly gained momentum in the 1970s [1]. Since then, numerous studies have compared TEWL in Atopic Dermatitis (AD) infants' clear and affected areas of the skin, and conducted chronological observation of TWEL levels in infants with $\mathrm{AD}$ and comparison with a control group to assess the effects of skincare preparations and products [2-5]. AD, which involves the elevation of the SC's barrier function indicator TEWL, is one of the allergy diseases that are on the increase. A large percentage of the cases manifest in infancy. Suspected aggravating factors include perspiration, poor physical condition, climate, sunlight, mental stress, fatigue, curettage, and food allergy. There are not many studies scrutinizing TEWL in healthy children in the $21^{\text {st }}$ century. The quantitative in-vivo measurement of SC's barrier function examines a minute amount of moisture transpiring through the SC. In other words, trans epidermal loss of moisture through the SC (TEWL) is measured in a cool, perspiration-free environment of infants living in a condition of minimal perspiration. A basic study has been carried out on the TEWL of infants living in urban and non-urban areas. This paper reports TEWL and SC water content of infant living in urban and non-urban areas as well as their environmental factors, measured at the same time.

\section{Methods}

The study covered infants aged 1-6 years old who attend childcare centres in the urban Area A and non -urban Area B.

\section{Measurement and investigation of the children's skin conditions}

Portable moisture transpiration gauge called VapoMeter (SWL04001) was used to measure the subjects' TEWL three consecutive times at the centre of the volar forearm. The median value of the three measurements was used as analysis data. The instrument also gave readings for outdoor air temperature, outdoor air humidity and surface temperature while measuring TEWL.

Portable skin moisture gauge called Moisture Meter (SC Compact) was used to measure the subjects' water content in the SC twice at the centre of the volar forearm. The higher of the two results was used as analysis data.

\section{Data gathering method}

The author contacted the department in charge of childcare centres at the selected areas' local councils to seek cooperation with this research. The childcare centres were asked to distribute and to collect research description, consent forms, and questionnaires on demographics such as age and gender of children to parents of the infants. The subjects were the returned consent forms of the parents for having their children's TEWL and SC water content measured as well as completed survey questionnaires. The first survey was conducted in early summer (June-July) and the second survey was conducted in autumn (mid-October).

\section{Analysis method}

Data was analysed with IBM Statistics 19.0 for descriptive statistics, Pearson product-moment correlation, t-test and chisquare test.

\section{Ethical considerations}

The children's parents were provided with written explanation about, and expressed consent in writing to how the study is based on the subjects' free will to participate, respects their decisions to withdraw from or continue with the study, protects 
their privacy and personal information, and has a defined scope and protocols for data usage. When distributing survey questionnaires, we enclosed written description about the measurement of skin conditions and a consent form. Since it is the infants who are subject to the measurement of skin conditions, the parents were asked to use the description, written in plain language, to explain (read) the information to their children and, based on the children's response, determine whether to consent to participating in the study, before filling out the consent form and sending it back. Those who responded to the survey questionnaires and provided a written, signed consent to participation in the measurement of skin conditions, were selected as the study's subjects. In the second survey, the subjects in the first survey were asked to participate again, and those who responded to the survey questionnaires and provided a written, signed consent to participation in the second measurement of skin conditions, were selected as the subjects. On the day of measuring skin conditions, the researchers and childcare workers accompanied the children, waiting for their turn, as they were shown photographs of a doll having the measurement taken and enlarged view of the measurement instruments used. Their response was observed to gauge whether they had a specific image about the measurement and how they felt about it, before the researchers checked if they could position their arm on the measurement table or required assistance. Prior to the measurement, approval was obtained from the author's organization's Research Ethics Review Board.

\section{Results}

\section{Attributes of the subjects}

Table 1 shows the subjects' attributes. The survey materials were sent initially to a total of 807 people (454 in Area A and 353 in Area B), and the second survey analyzed results of 523 people (275 in Area A and 248 in Area B) (effective response rate of $64.8 \%$ ). The subjects were broken down into 281 males and 242 female children, with no significant difference in gender ratios between the two areas. The average age of all the children was 44.3 months, with no significant difference between the two areas (43.6 months for Area A and 45 months for Area B).

\section{TEWL, SC water content and environmental factors for all subjects}

Table 2 showed the average results of two measurements in TEWL, SC water content and environmental factors for all the subjects. The average TEWL of infant was $16.7 \pm 8.9 \mathrm{~g} / \mathrm{m}^{2} / \mathrm{h}$ in the first measurement (June- July) and $14.7 \pm 6.4 \mathrm{~g} / \mathrm{m}^{2} / \mathrm{h}$ in the second measurement (October). The SC water content was $34.1 \pm 20.7 \mathrm{AU}$ in the first measurement and 16.7 $\pm 10.0 \mathrm{AU}$ in the second measurement. There were significant differences between the two measurements in TEWL, SC water content, surface temperature, outdoor humidity and outdoor temperature. There was also significant positive correlation between TEWL from the two measurements (Figure 1).

Table 3 shows the average results of two measurements in TEWL, SC water content and environmental factors, broken down by area. In the first measurement, Area B showed significantly higher results than Area A in TEWL, outdoor temperature, surface temperature and SC water content. The exception was the outdoor humidity, which was signficantly lower in Area B than in Area A. In the second measurement, there was no signiicant difference in outdoor air temperature between two areas, but TEWL,SC water content and outdoor humidity were signficantly higher in Area B than in Area A.

Figure 2 is a scatter diagram of TEWL and SC water content. For both measurements, TEWL and SC water content had a significant positive correlation.

Table 1. Subjects.

\begin{tabular}{|c|c|c|c|}
\hline & Total & Area A(urban) & $\begin{array}{c}\text { Area B } \\
\text { (non-urban) }\end{array}$ \\
\hline Number of Subjects & 523 & 275 & 248 \\
\hline Gender: Male & 281 & 152 & 129 \\
\hline Female & 242 & 123 & 119 \\
\hline Average age in months & $44.3 \pm 16.8$ & $43.6 \pm 16.5$ & $45.0 \pm 17.2$ \\
\hline *No significant difference between the two areas \\
\hline
\end{tabular}

Table 2. Average values of TEWL, SC water content and environmental factors.

\begin{tabular}{|c|c|c|}
\hline & $\begin{array}{l}1^{\text {st }} \text { measurement } \\
\text { June - July }\end{array}$ & $\begin{array}{c}2^{\text {nd }} \text { measurement } \\
\text { October }\end{array}$ \\
\hline TEWL $\left(\mathrm{g} / \mathrm{m}^{2} / \mathrm{h}\right)$ & $16.7 \pm 8.9^{\star *}$ & $14.7 \pm 6.4$ \\
\hline SC water content & $34.1 \pm 20.7^{* *}$ & $16.7 \pm 10.0$ \\
\hline Outdoor humidity & $53.5 \pm 7.8^{* \star}$ & $44.7 \pm 5.3$ \\
\hline Outdoor temperature & $28.6 \pm 1.2^{* *}$ & $26.7 \pm 1.4$ \\
\hline Surface temperature & $33.2 \pm 2.0^{* *}$ & $32.2 \pm 1.8$ \\
\hline
\end{tabular}

Table 3. Average TEWL, SC water content and environmental factors for the two areas.

\begin{tabular}{|c|c|c|c|c|}
\hline & \multicolumn{2}{|c|}{ June-July } & \multicolumn{2}{c|}{ October } \\
\cline { 2 - 5 } & Area B & Area A & Area B & Area A \\
\hline TEWL $\left(\mathrm{g} / \mathrm{m}^{2} / \mathrm{h}\right)$ & $18.3^{* *}$ & 15.4 & $15.5^{* *}$ & 13.9 \\
\hline SC water content & $41.8^{* *}$ & 27.1 & $18.1^{* *}$ & 15.4 \\
\hline Outdoor humidity & 52.3 & $54.6^{* *}$ & $45.5^{\star *}$ & 44.1 \\
\hline Outdoor temperature & $29.5^{* *}$ & 28.2 & 26.7 & 26.7 \\
\hline Surface temperature & $33.8^{* *}$ & 32.6 & $32.8^{* *}$ & 31.6 \\
\hline & & & \multicolumn{2}{|c|}{${ }^{*} \mathrm{p}<0.05 ;{ }^{* *} \mathrm{p}<0.01$} \\
\hline
\end{tabular}

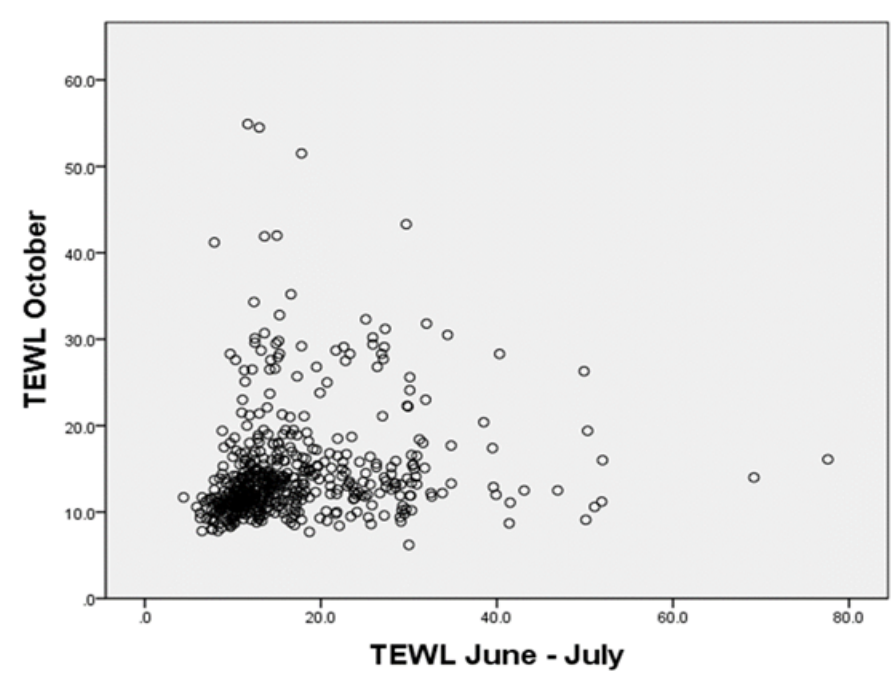

Figure 1. Correlation of two of the timing of TEWL of infant. Significant positive correlation $(p<0.01)$ was shown in the two values. 


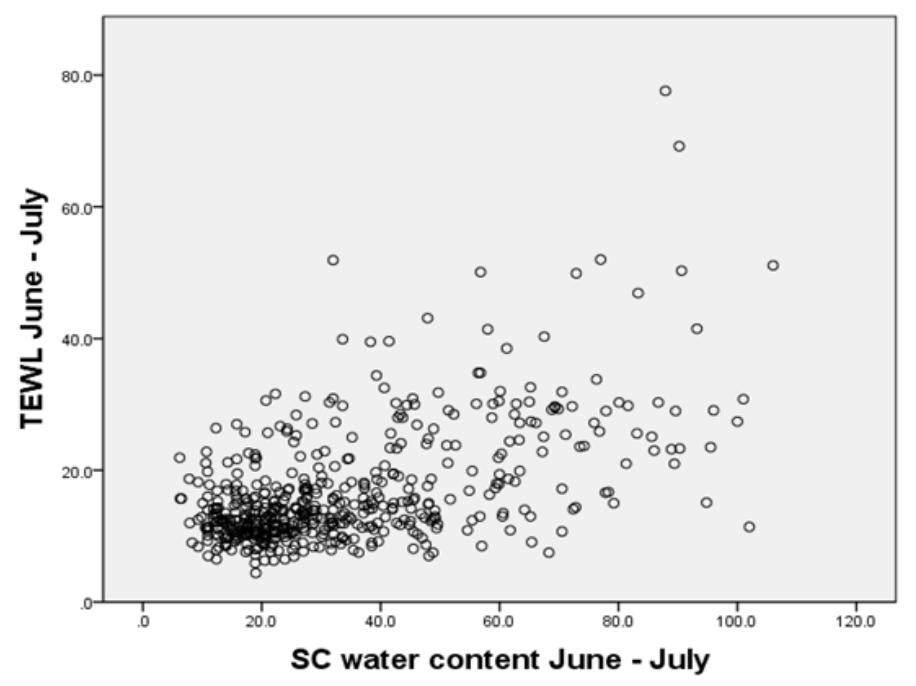

(a)

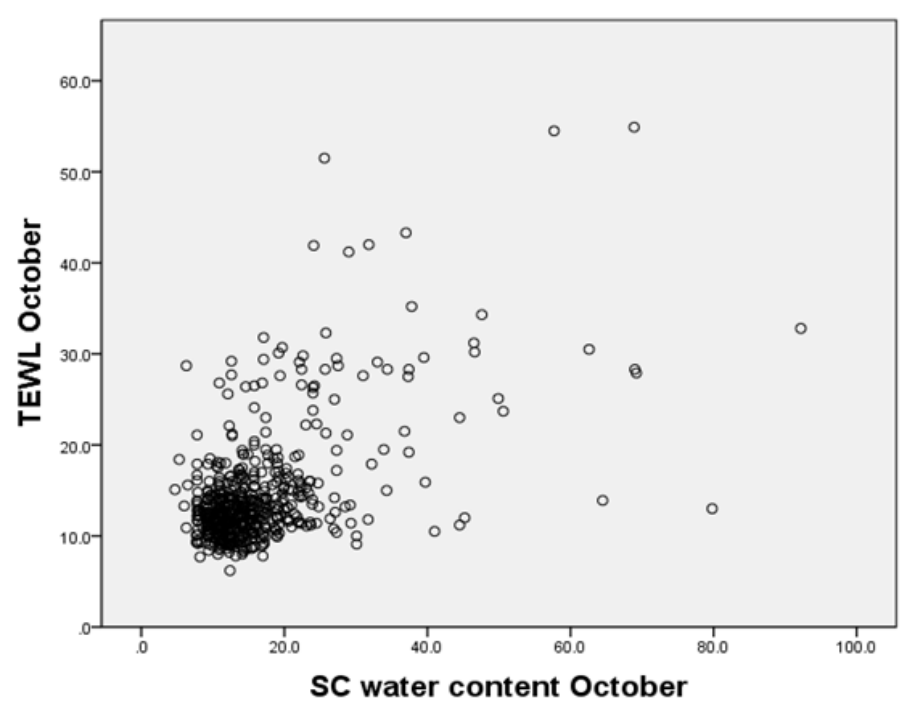

(b)

Figure 2. (a) Correlation between TEWL and SC water content was measured in June-July. Significant positive correlation $(p<0.01)$ was shown in the two values. (b) Correlation between TEWL and SC water content was measured in October. Significant positive correlation ( $p$ $<0.01)$ was shown in the two values.

\section{TEWL and SC water content by gender}

Table 4 shows the gender breakdown of average TEWL and SC water content among all the subjects. In both measurements, TEWL for male subjects was significantly higher than that for female subjects. There was no significant gender difference in $\mathrm{SC}$ water content for both measurements.

Table 4. Average TEWL and SC water content by gender.

\begin{tabular}{|c|c|c|c|}
\hline \multicolumn{2}{|c|}{} & Male (n=281) & Female (n=242) \\
\hline \multirow{2}{*}{ TEWL } & June-July & $17.7 \pm 9.1^{* *}$ & $15.6 \pm 8.6$ \\
\cline { 2 - 4 } & October & $15.7 \pm 8.6^{* *}$ & $13.5 \pm 5.4$ \\
\hline \multirow{2}{*}{$\begin{array}{c}\text { SC water } \\
\text { content }\end{array}$} & June-July & $35.4 \pm 20.9$ & $32.6 \pm 20.3$ \\
\cline { 2 - 4 } & October & $17.4 \pm 9.2$ & $15.9 \pm 10.719 .7$ \\
\hline
\end{tabular}

\section{TEWL and SC water content in three infancy stages}

Table 5 shows the average TEWL and SC water content in the two measurements. The development of infant is broken down into three stages; Stage I (1-2 years old), Stage II (3-4 years old) and Stage III (5-6 years old). In the two measurements, TEWL showed a significant difference between Stage I and Stage II of infancy, with Stage II infancy having a significantly higher result than Stage I. In measurement from June to July, there was a significant difference in TEWL between Stage I and Stage III infancy.

No significant difference was observed between Stage II and Stage III infancy in both measurements. The fluctuations in TEWL between the two measurements were smaller in latter stages of infancy as the infants became older. As for SC water content, there was a significant difference between the three infancy stages in both measurements. However, the two measurements of the SC water content showed no significant difference between each of the infancy stages.

Figure 3 is a scatter diagram of age (month) and TEWL in two measurements of TEWL. There was a significant negative correlation only for the June-July measurement.

\section{Discussion}

\section{TEWL and SC water content, broken down by age and gender}

In this study, the average TEWL of infants at the volar forearm was $16.7 \pm 8.9 \mathrm{~g} / \mathrm{m}^{2} / \mathrm{h}$ (June-July) and $14.7 \pm 6.4 \mathrm{~g} /$ $\mathrm{m}^{2} / \mathrm{h}$ (October). We found that the smaller the age, the larger the value range of TEWL in the seasonal difference between June-July at 28.6 degrees Celsius in average temperature and $53.6 \%$ in average humidity and October at 26.7 degrees Celsius in average temperature and $44.7 \%$ in average humidity. TEWL values from the upper ventral arm is higher for the first 12 months of life compared to adult [1]. The moisture transpiration in infants (average age of $16.1 \pm 5.2$ months) was $21.0 \pm 9.3 \mathrm{~g} /$ $\mathrm{m}^{2} / \mathrm{h}$, significantly higher than that of adults $\left(11.8 \pm 5.7 \mathrm{~g} / \mathrm{m}^{2} / \mathrm{h}\right)$ [6]. The two measurements of TEWL in this study were lower than the moisture transpiration volume of infants (average age of $16.1 \pm 5.2$ months $)\left(21.0 \pm 9.3 \mathrm{~g} / \mathrm{m}^{2} / \mathrm{h}\right)$ and higher than that of adults $\left(11.8 \pm 5.7 \mathrm{~g} / \mathrm{m}^{2} / \mathrm{h}\right)$. The properties that make infant skin unique continue to persist at least through the first 12 months of life. Infant barrier function and water-handling properties of $\mathrm{SC}$ continue to be in a state of flux until more stable regulation

Table 5. TEWL and SC water content in three infancy stages. $(n=523)$

\begin{tabular}{|c|c|c|c|c|}
\hline Three Stages & Item & $\begin{array}{l}\text { 1st } \\
\text { measurem } \\
\text { June-July }\end{array}$ & & $\begin{array}{l}\text { 2nd } \\
\text { measurement } \\
\text { October }\end{array}$ \\
\hline Stage I infancy & TEWL & 19.7 & $\ldots * *$ & 15.7 \\
\hline $\begin{array}{c}\text { (1-2 years old) } \\
\quad n=181\end{array}$ & SC water content & $34.6 \quad * *$ & $* *$ & $17.3^{* *}$ \\
\hline Stage II infancy & TEWL & $15.1 \longrightarrow$ & $*$ & $13.6-1$ \\
\hline $\begin{array}{c}\text { (3-4 years old) } \\
n=214\end{array}$ & & 34.4 & $\begin{array}{r} \\
* * \\
* * \ldots \ldots .\end{array}$ & 36.0 \\
\hline $\begin{array}{l}\text { Stage II infancy } \\
\begin{array}{c}\text { (5-6 years old) } \\
n=128\end{array}\end{array}$ & $\begin{array}{l}\text { TEWL } \\
\text { SC water content }\end{array}$ & $\begin{array}{l}15.3 \\
32.9\end{array}$ & + & $\begin{array}{r}14.9 \\
16.1 \\
\ldots \ldots . .\end{array}$ \\
\hline
\end{tabular}




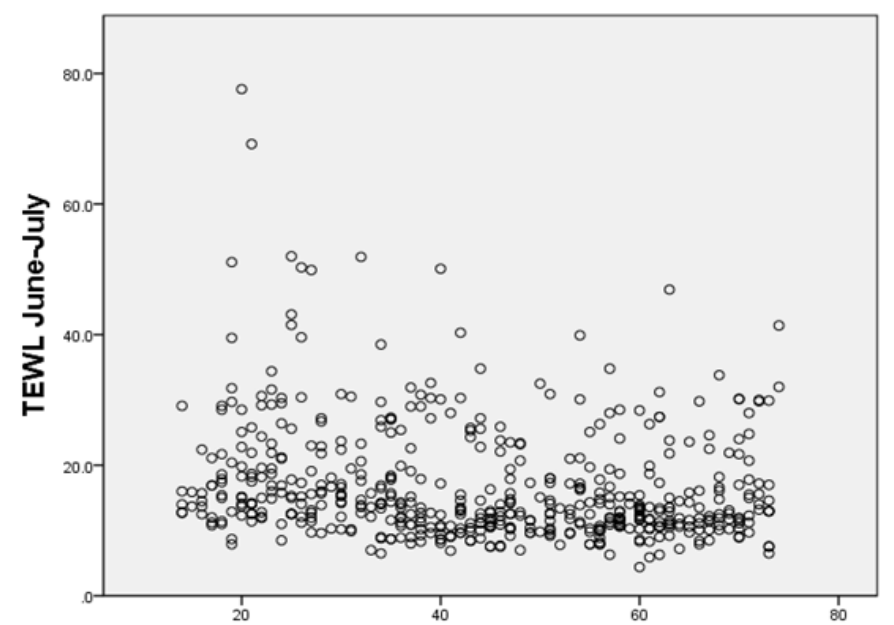

Age(month) of child when measured in June - July

(a)

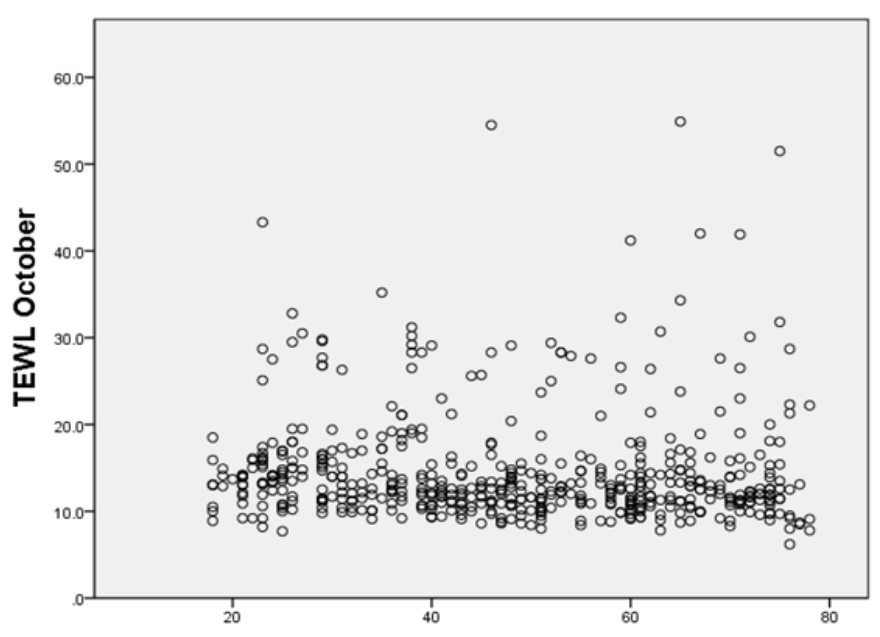

Age (month) of child when measured in October

(b)

Figure 3. (a) Correlation of infant age and TEWL in June-July. Significant negative correlation $(p<0.01)$ was shown in the two values. (b) Correlation of infant age and TEWL in October. No significant difference between the two values.

mechanisms are developed later in life [7]. Infants' TEWL could be improving the epidermal barrier function by fluidly reducing the range of fluctuations according to room temperature, humidity, season, etc. as infants grow older in order to obtain a mechanism of greater stability.

In this study, the SC water content of infants' volar forearm was $34.1 \pm 20.7 \mathrm{AU}$ in the first measurement (June-July at 28.6 degrees Celsius in average temperature and $53.6 \%$ in average humidity) and $16.7 \pm 10.0 \mathrm{AU}$ temperature and $44.7 \%$ in average humidity). Infants (average age of $16.1 \pm 5.2$ months) had the same level of water content ( $42.8 \pm 12.0$ A.U.) as adults (39.0 \pm 9.7 A.U.) [6]. This suggests that infants' SC water content in the first measurement was about the same level as adults' SC water content. Since the environmental factors surrounding the skin, e.g. low humidity, low temperature, air current, etc., are known to reduce $\mathrm{SC}$ water content on the surface of even healthy skin, the infants' $\mathrm{SC}$ water content in the second measurement is suspected to have been lower than in the first measurement in the case of seasonal fluctuation caused by lower room temperature and humidity [8].

The two measurements in this study indicated a significant positive correlation between SC water content and TEWL. There is no such correlation between SC water content and TEWL for those with healthy skin. However, for people with Atopic Dermatitis (AD), there is a significant negative correlation between SC water content and TEWL, shown in a correlation diagram [9]. The mechanism behind it is explained as the increase of Transepidermal Water Loss (TEWL) reducing SC water content, causing the skin to dry [10]. The results of this study did not substantiate the findings of past studies. It is unknown whether this was due to differences in measurement environment/equipment, or the fluidity of healthy infants' water retention/epidermal barrier mechanisms. Healthy infants' SC water content could affect their TEWL, which should be explored further.

This study has shown that male infants have a significantly higher TEWL than female infants do. There was no significant genderbased difference in infants' SC water content. The TEWL of the men from the 20-year-old to 50-year-old is significantly lower than the water loss of women of the same age group, regardless of the location. With ageing, gender-related differences in TEWL assimilate. Young men show higher SC water content in comparison with women [11]. In other words, genderbased tendency in TEWL and SC water content among adults contradicted the findings of this study. Further examination of TEWL is needed with different measurement environment, increased subject count and broader range of subjects' ages to cover school-age and adolescent children.

\section{TEWL, SC water content and environmental factors}

This study has shown significant difference in infants' TEWL, $\mathrm{SC}$ water content and surface temperature between the two measurement timings, with the results from early summer (JuneJuly) significantly higher than those from autumn (October). This means that infants' water retention function in SC deteriorates in autumn compared to early summer, while their epidermal barrier function lowers in early summer compared to autumn. Elements contributing to SC's water retention function include intercellular lipid, sebum, lactic acid/urea components of sweat and hyaluronic acid generated on the epidermis. This is why children and elderly persons with low sebum secretion are prone to dry skin in winter [12]. In Japan, compared to spring-born babies who grow up in warm and humid conditions, autumn-born babies who grow up in cold and dry conditions tend to suffer from dry skin, with some developing a mild case of AD [13]. This was considered to be the reason infants' SC water content was lower in autumn than in early summer. In this study, the $\mathrm{SC}$ water content was high in early summer when TEWL also increased. When the skin's inside-to-outside barrier function is compromised, the TEWL from the skin surface increases, thereby reducing the SC water content and triggering dry skin [10]. For this reason, this study's subjects have a low risk of developing dry skin and do not show the deterioration of the epidermal barrier function to the extent of triggering skin problems. Since the level of surface temperature was to have 
retained a large amount of water in the SC during summer, and transpired a large amount of water from the epidermis through physical activities and perspiration, keeping the skin's functions to retain water content, perspire and control the body temperature active. There are some reports suggesting that the amount of ceramide in intercellular lipid, one of the factors contributing SC's water retention performance, is lower in those suffering from $\mathrm{AD}$, and that the amount of ceramide has correlation with TEWL. However, not many of these reports directly establish connection with the SC's water retention performance, and the SC water content could be affected collectively by the functionality of not only the epidermis but also sweat glands and sebum. The SC's mechanism of water retention remains largely unexplained [9]. This is why the results of this study must be interpreted with caution.

\section{TEWL, SC water content and measurement methods}

This study measured infants (aged 1-6 with the average of 3 Y $8 \mathrm{M}$ ) volar forearm TEWL in early summer (Average 16.7 $\pm 8.9 \mathrm{~g}$ / $\mathrm{m}^{2} / \mathrm{h}$ ) and autumn (Average $14.7 \pm 6.4 \mathrm{~g} / \mathrm{m}^{2} / \mathrm{h}$ ). In similar past studies, no significant differences in TEWL were found between infants and adults, either on the buttocks or on the volar forearm [2]. TEWL of healthy adults, measured in a cool, perspirationfree environment of around 20 degrees Celsius, was around $5 \mathrm{~g} /$ $\mathrm{m}^{2} / \mathrm{h}$ at the body trunk and limbs and over $10 \mathrm{~g} / \mathrm{m}^{2} / \mathrm{h}$ in the face, which has thinner SC [13]. TEWL of 1-2 year olds (average $\mathrm{TEWL}=9.79 \mathrm{~g} / \mathrm{m}^{2} / \mathrm{h}$ ) and 4-5 year olds (average TEWL=7.2 g/ $\mathrm{m}^{2} / \mathrm{h}$ ), measured at their volar forearm in the environments of 20 \pm 2 degrees Celsius and $50 \pm 10 \%$, had no significant difference from TEWL of $20-35$ year olds $\left(8.9 \mathrm{~g} / \mathrm{m}^{2} / \mathrm{h}\right)$ [14]. TEWL of infants aged 38 months or above, measured at the abdomen, thigh and buttocks was similar to that of their mothers' [6]. As for school- age and adolescent children, TEWL of 10-14-yearold children (measured at the forearm) was significantly higher than that of adults in both summer and winter [15]. In TEWL measurement, it is difficult to have uniform measurement conditions. This is why measurements are taken in multiple locations and timings, or carried out on subjects of different developmental stages. Future studies should consider taking measurements at multiple locations and covering subjects of different developmental stages such as school-age children [16].

\section{TEWL, SC water content and areas of residence}

Infants' TEWL, SC water content and surface temperature were signiicantly higher for subjects in the non-urban area than those in the urban area. The criteria for selecting the survey areas in this survey were the population size, population density, opposite tendency in juvenile (15 years old or younger) population density and location (in the Honshu main island of Japan). Area A has a high population, high population density and low juvenile (15 years old or younger) population density, with children growing up surrounded by many adults. Area B has a low population, low population density but high juvenile (15 years old or younger) population density, with many children growing up with other children and adults. No past study reporting the effect of infants' living environment (e.g. different areas of residence) on their skin was found. However, some studies reported that the lower the room temperature is, the lower the SC water content's indicators become, including conductance, TEWL, blood flow volume and the skin's surface temperature [8]. In the comparison between long-term bedridden seniors and healthy subjects, the bedridden patients had a higher ratio of those suffering from dry skin and skin atrophy, and demonstrated significant differences in TEWL, SC water content and dermal $\mathrm{pH}$ [17]. A study indicated that the high TEWL values shown for some subjects may be indicative of a mildly stressed subject rather than suggestive of a sustained high-water flux [7]. These reports show that it is necessary to examine not only external environmental factors but also living environment factors, lifestyles and stress, which could affect epidermis and SC, in future studies on children [18].

\section{Conclusion}

The two measurements of infants' TEWL in two areas found the following:

- Infants' TEWL was significantly higher in early summer (June-July) than in autumn (October).

- Infants in early stage of development had higher TEWL than infants in middle or late stage of development.

- Seasonal difference in infants' TEWL became smaller as they advanced through their developmental stages.

- Male infants' TEWL was significantly higher than that of female infants.

- Infants' SC water content showed no significant difference according to their developmental stages or gender, but indicated a significant seasonal difference (higher in early summer than in autumn).

- Infants' who live in the non-urban area (Area B) have a significantly higher surface temperature on the skin than infants who live in an urban area (Area A), retaining more moisture in the $\mathrm{SC}$ and transpiring more moisture from the epidermis.

\section{Acknowledgements}

This work was supported by JSPS KAKENHI Grant Number JP22659418, JP16K15938.

I wish to acknowledge the cooperation and the support of all people who participated in this research.

\section{Conflict of Interest}

The author has no conflicts of interest directly relevant to this article.

\section{References}

1. Tagami H. Horny layer as a barrier to the skin. Jpn J Dermatol. 1998;108:713-27.

2. Giusti F, Martella A, Bertoni L, et al. skin Barria hydration, and $\mathrm{pH}$ of the skin of infants under 2 years of age. Pediatr Dermatol. 2001;18:93-6.

3. Chamlin SL, Kao J, Frieden IJ. Ceramide-dominant barrier 
Citation: Ono S, Manabe Y. Basic study on Transepidermal Water Loss (TEWL) of infants living in urban and non-urban areas and their environmental factors. J Preg Neonatal Med. 2017;1(1):1-6.

repair lipids alleviate childhood atopic dermatitis: Changes in barrier function provide a sensitive indicator of disease activity. J Am Acad Dermatol. 2002;47:198-207.

4. Hon EK, Leung T, Ching G. Patterns of food and aeroallergen sensitization in childhood eczema. Acta Paediatr. 2008;97:1734-7.

5. Machado M, Hadgraft J, Lane ME. Assessment of the variation of skin barrier function with anatomic site, age, gender and ethnicity. Int J Cosmet Sci. 2010;32:397-409.

6. Miyauchi Y. Observation of infants' skin-dermal development. J Cosmet Sci. 2014;38:28-36.

7. Nikolovski J, Stamatas GN, Kollias N, et al. Barrier function and water-holding and transport properties of infant stratum corneum are different from adult and continue to develop through the first year of life. J Invest Dermatol. 2008; 128:1728-36.

8. Kikuchi K. Environment and dry skin-low humidity environment's impact on the skin. MB Derma. 2012;196:63-7.

9. Sakai S. Skin's moisture retention mechanism. MB Derma. 2012;196:9-16.

10. Akiyama M. Basic up-to- date knowledge on skin's barrier function. Jpn Int J Cosmet Sci. 2014;38:22-7.

11. Luebberding S, Krueger N, Kerscher M. Skin physiology in men and women: in vivo evaluation of 300 people including
TEWL, SC hydration, sebum content and skin surface $\mathrm{PH}$ Int J Cosmet Sci. 2013;35:477-83.

12. Tagami H. Atopic dermatitis patients' SC functions and the effects of treatment with moisturizer and topical steroid. Yakkyoku. 2013;64:63-72.

13. Tagami H. Xeroderma overview. MB Derma. 2012;196:1-8.

14. Fluhr, JW, Darlenski R, Lachmann N. Infant epidermal skin physiology: adaptation after birth. $\mathrm{Br} \mathrm{J}$ Dermatol. 2013;166:483-90.

15. Akutsu N, Ooguri M, Onodera T. Functional characteristics of the skin surface of children approaching puberty: age and seasonal influences. Acta Derm Venereol. 2009;89:21-7.

16. Kikuchi, Katsuko, Tagami H. Noninvasive biophysical assessments of the efficacy of a moisturizing cosmetic cream base for patients with atopic dermatitis during different seasons. Br J Dermatol 2008;158:969-78.

17. Hori R, Mizuguchi Y, Okamura N, et al. Skin's surface conditions, skin cleansing method and effects of skincare routine in long-term bedridden senior patients and healthy subjects. Niigata Nursing College Journal. 2012;1:17-23.

18. Kircik K, Tagami H. Transepidermal water loss (TEWL) and corneometry with hydrogel vehicle in the treatment of atopic dermatitis: a randomized, investigator-blind pilot study. J Drugs Dermatol. 2012;11:180-4.

\section{*Correspondence to:}

Satomi Ono

Department of Nursing

Graduate School of Health Science

Okayama University

Japan

Tel: +81-86-235-6868

E-mail: satomi-ono@okayama-u.ac.jp 